
for community-based electricity feedback

\title{
Smartphone 2010
}

Markus Weiss, Thorsten Staake, Friedemann Mattern, and Elgar Fleisch

Institute of Technology Management (ITEM), University of St. Gallen

Chair of Information Management (D-MTEC), ETH Zurich

Institute for Pervasive Computing (D-INFK), ETH Zurich

Gwangju / December 9-11, 2010

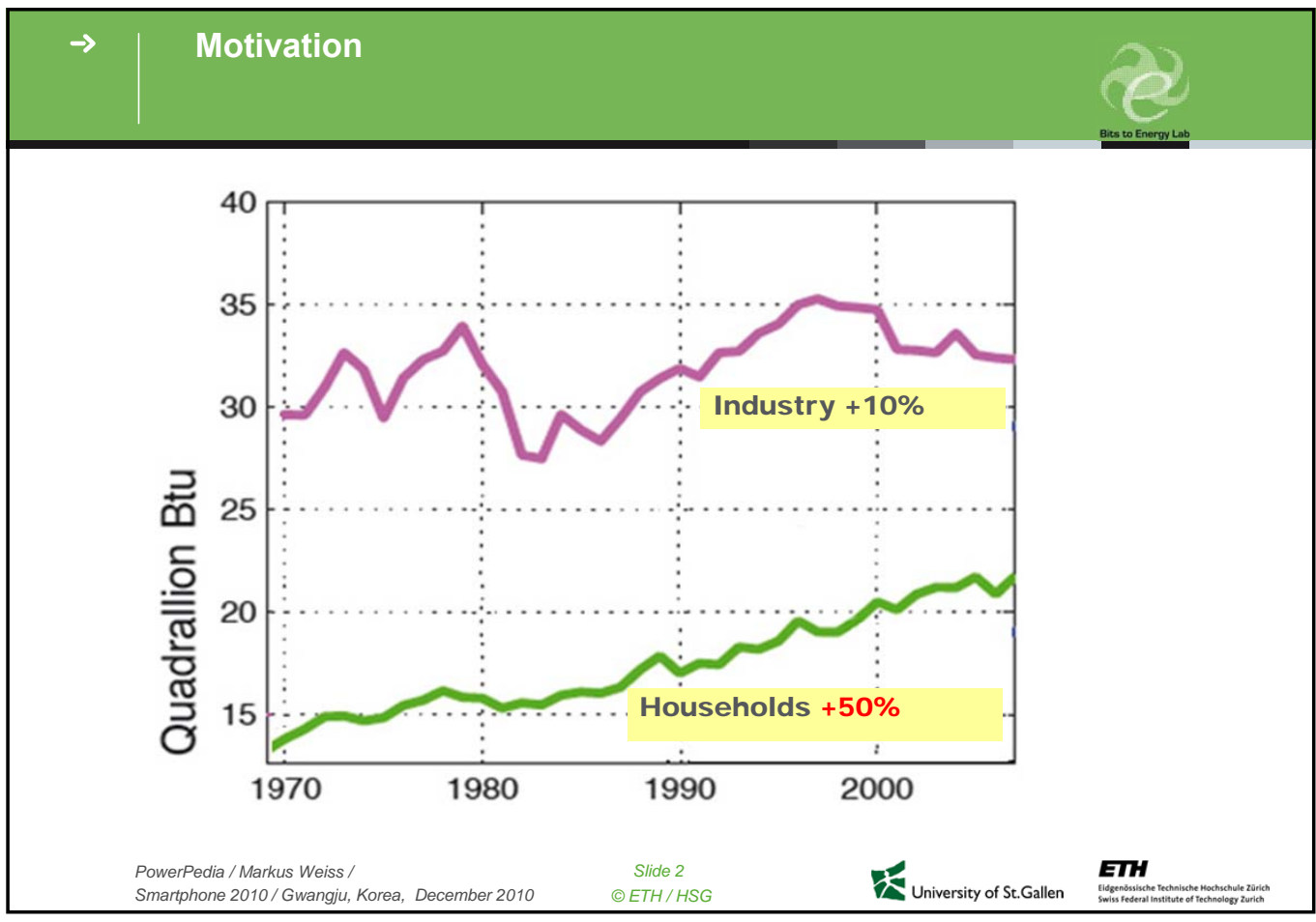



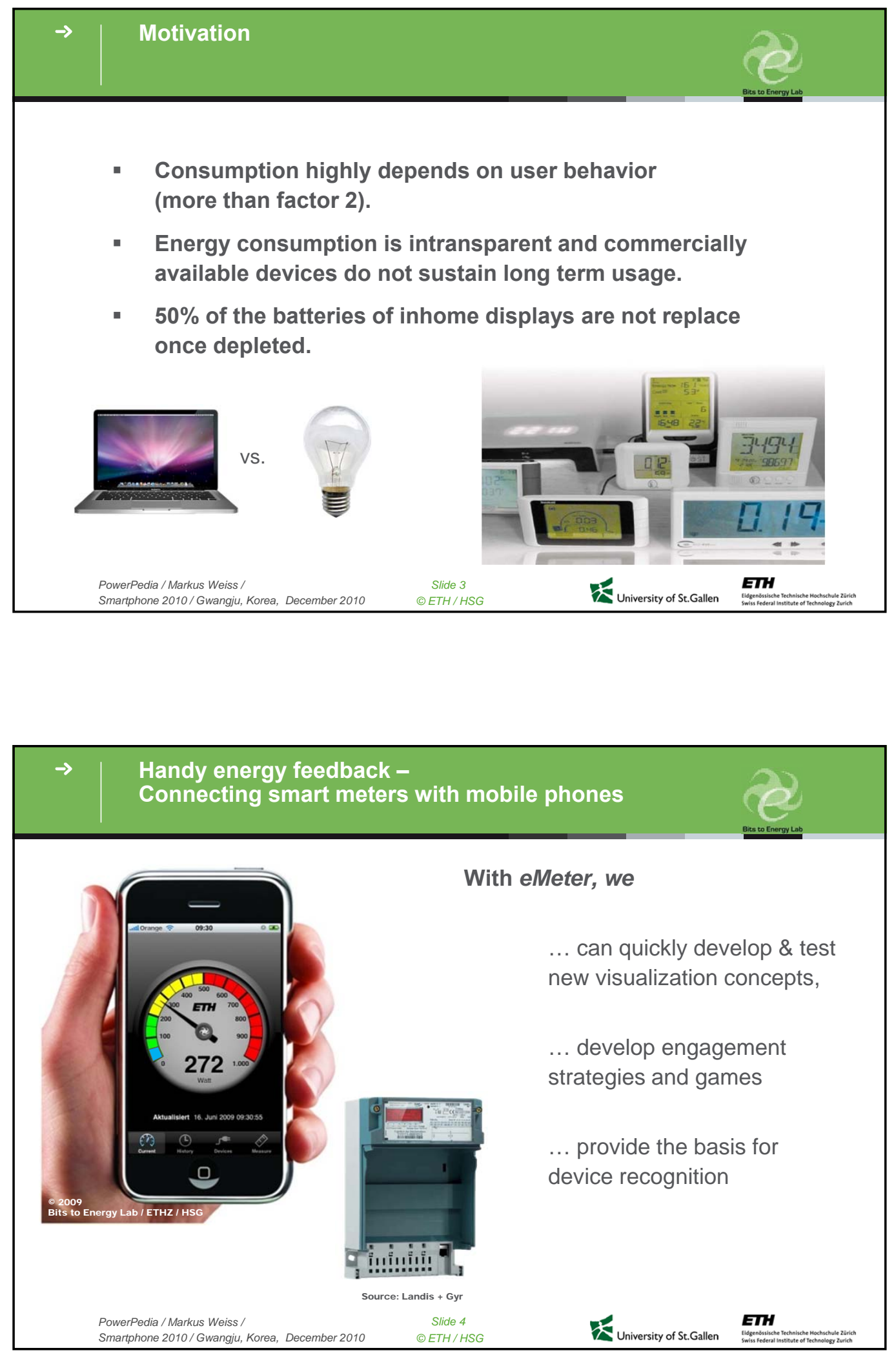

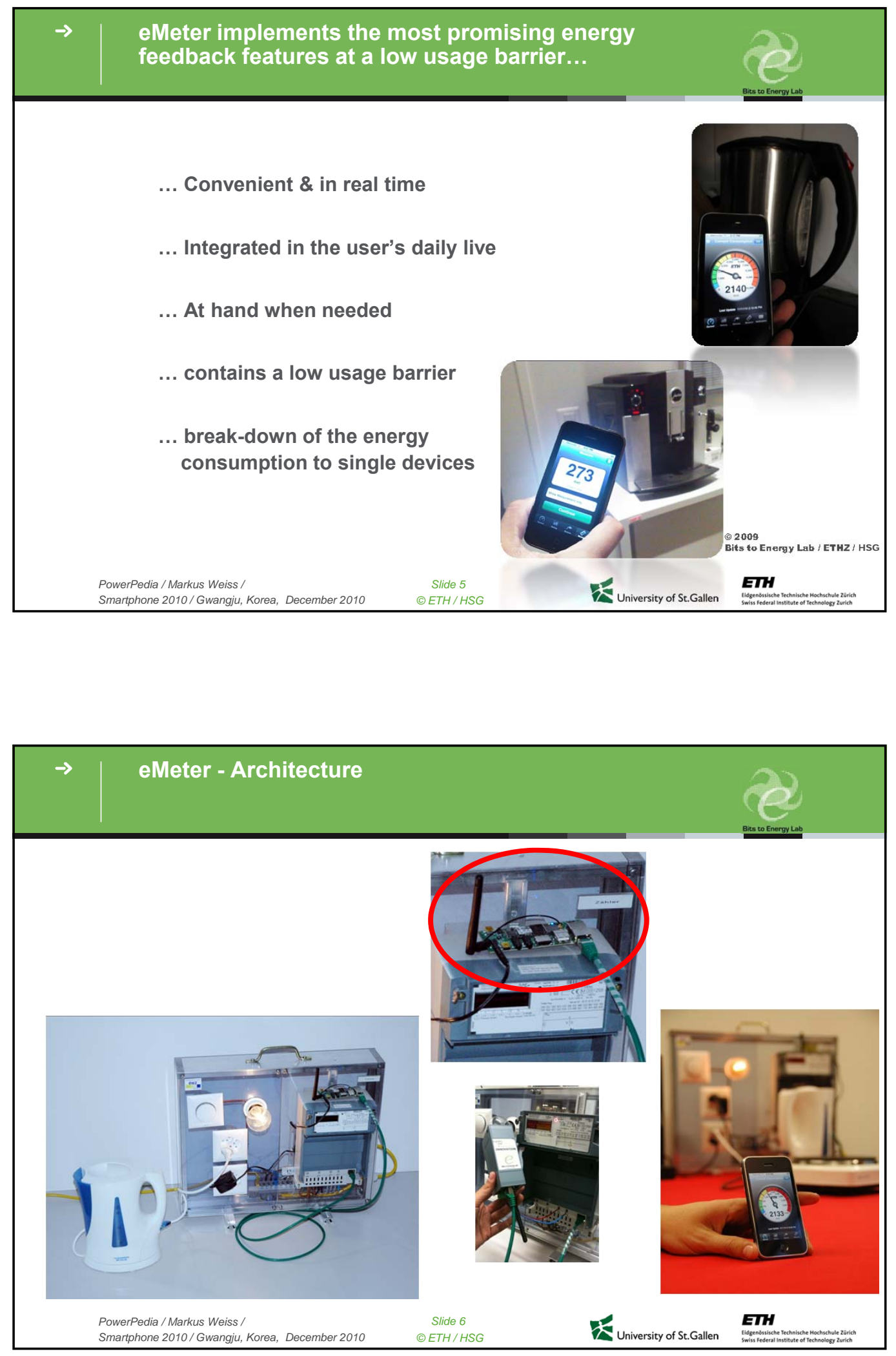


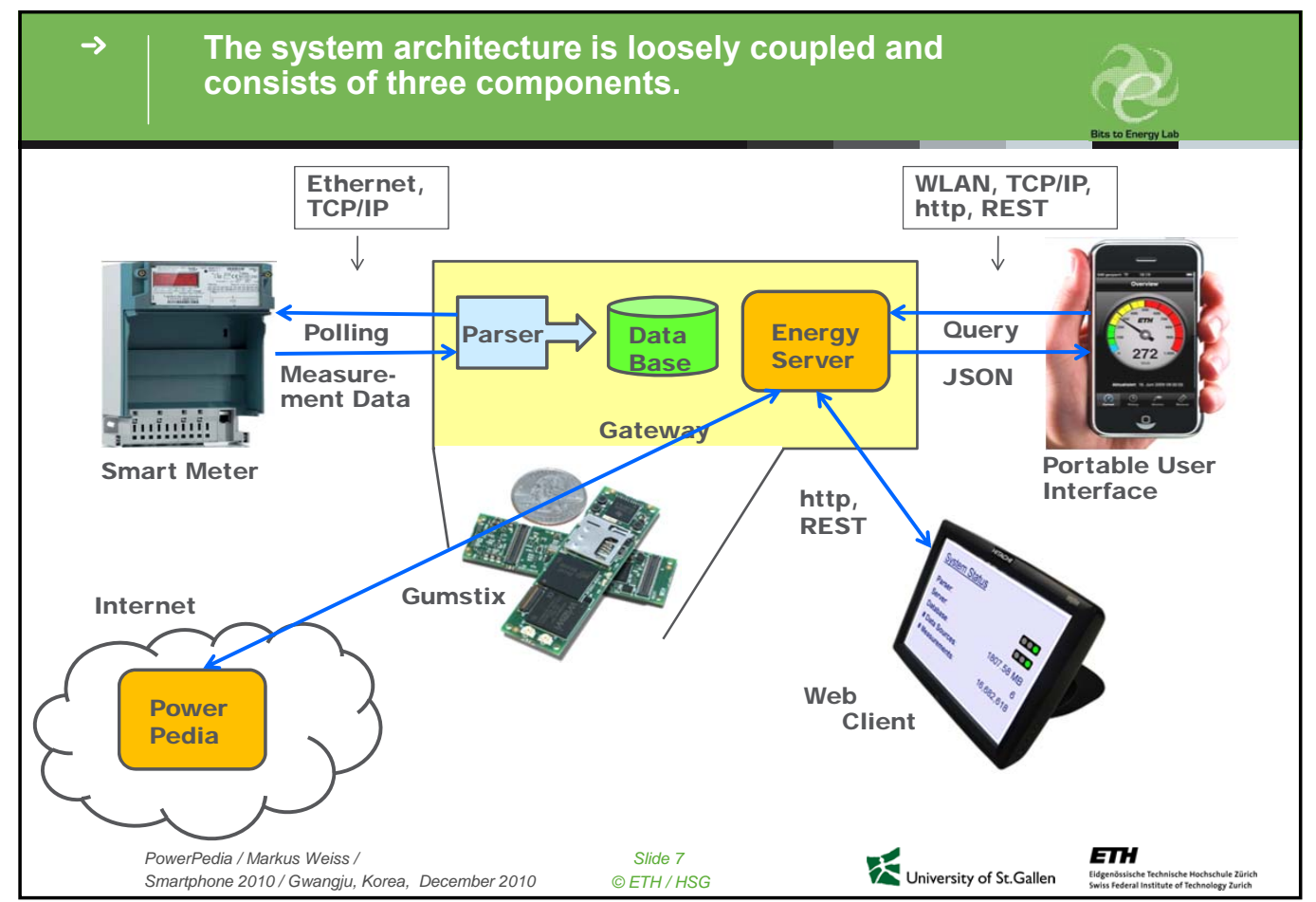

$\rightarrow \quad$ Using a restful approach guarantees high

interoperability, loose coupling, faster prototyping,

bigger flexibility, and robustness.

REST architectural style describes the following five constraints applied to the architecture

- Client-server

- Stateless

- Uniform interface

- Uses http commands

- Layered system

http verbs: Get, Put, Delete, and Post to access, update, delete, and create resources

http header: for metadata and status information

http body: the actual data 

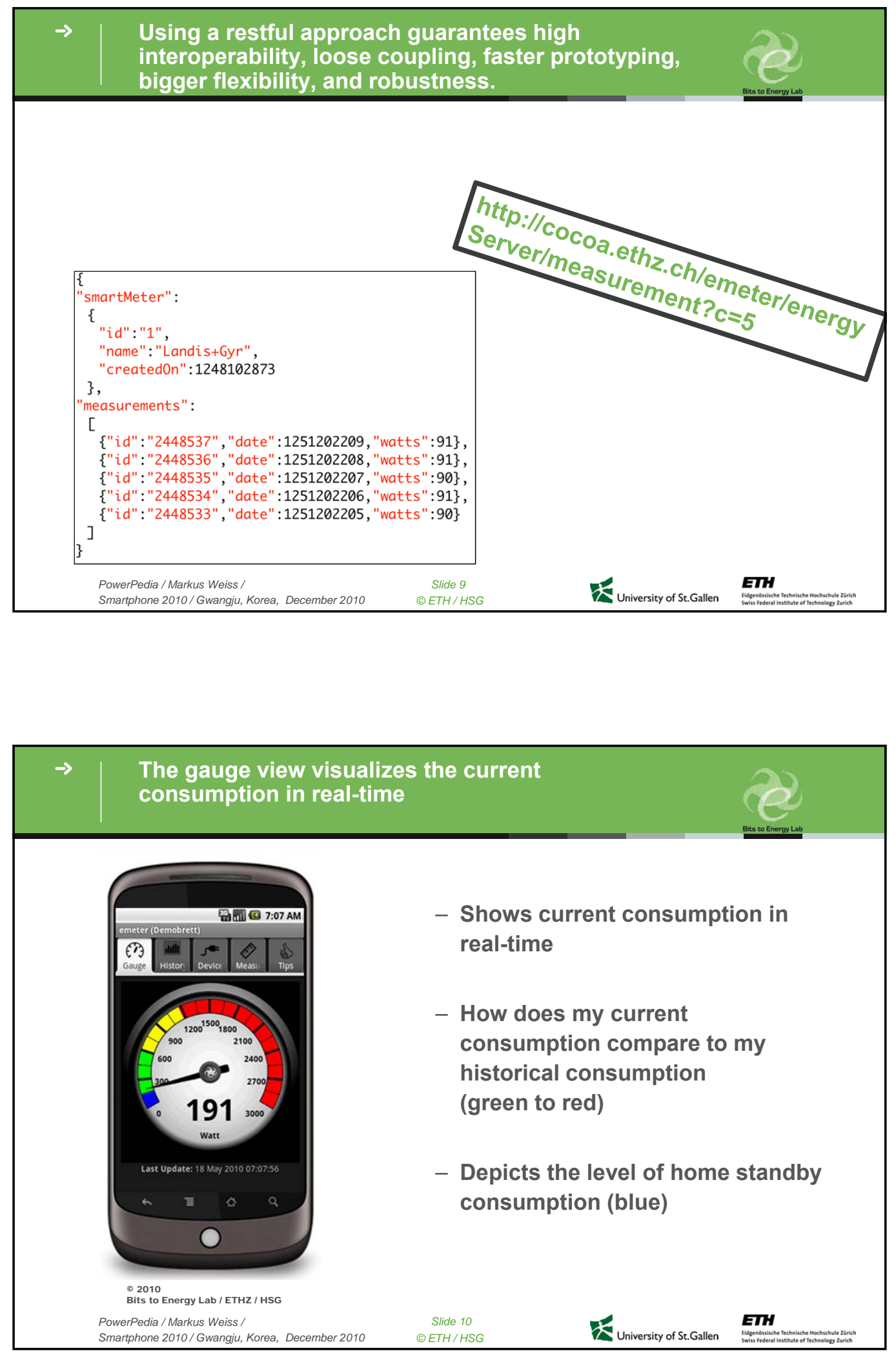


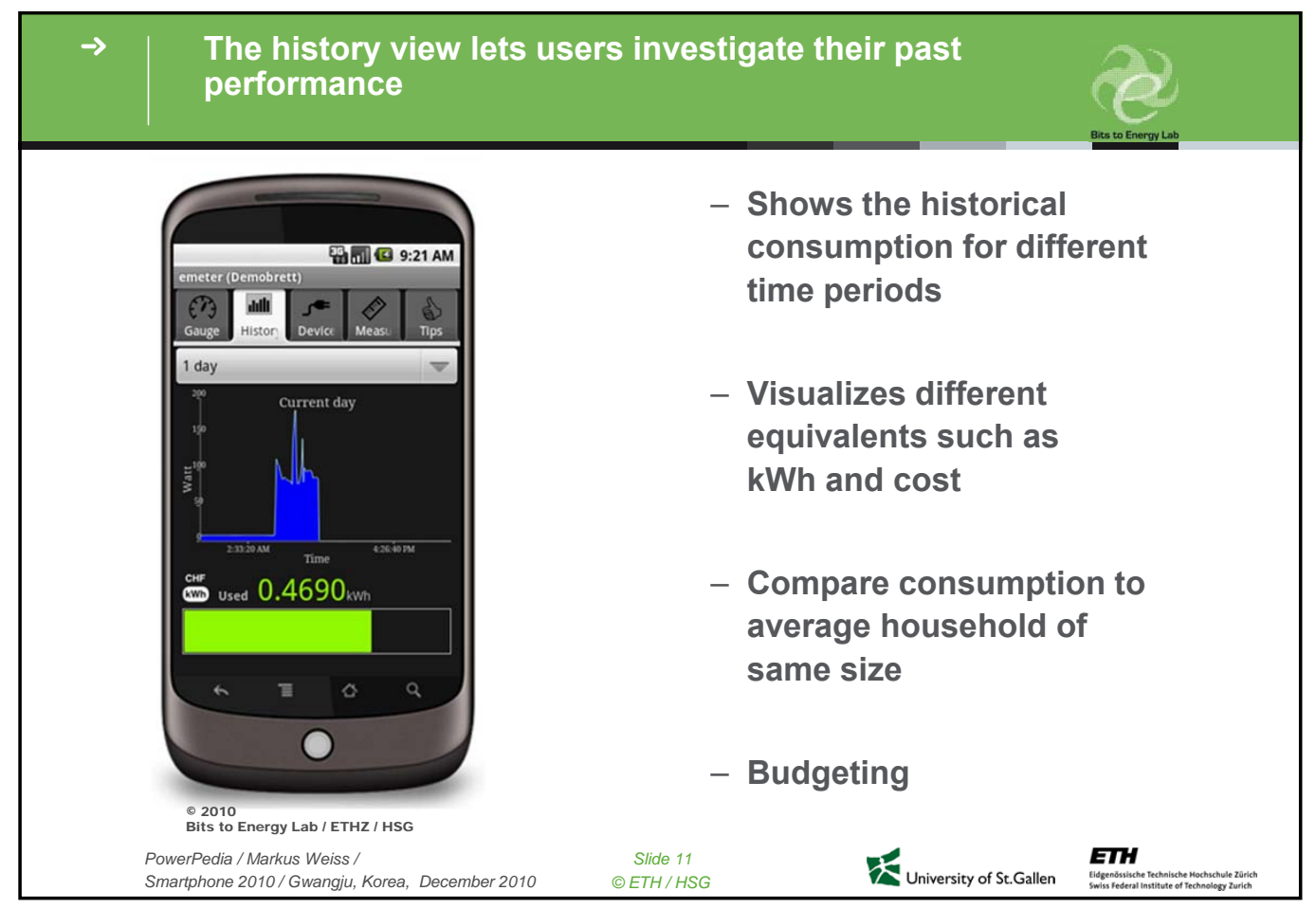

$\rightarrow \quad$ The measurement functionality allows users to interactively determine the power consumption of switchable devices.

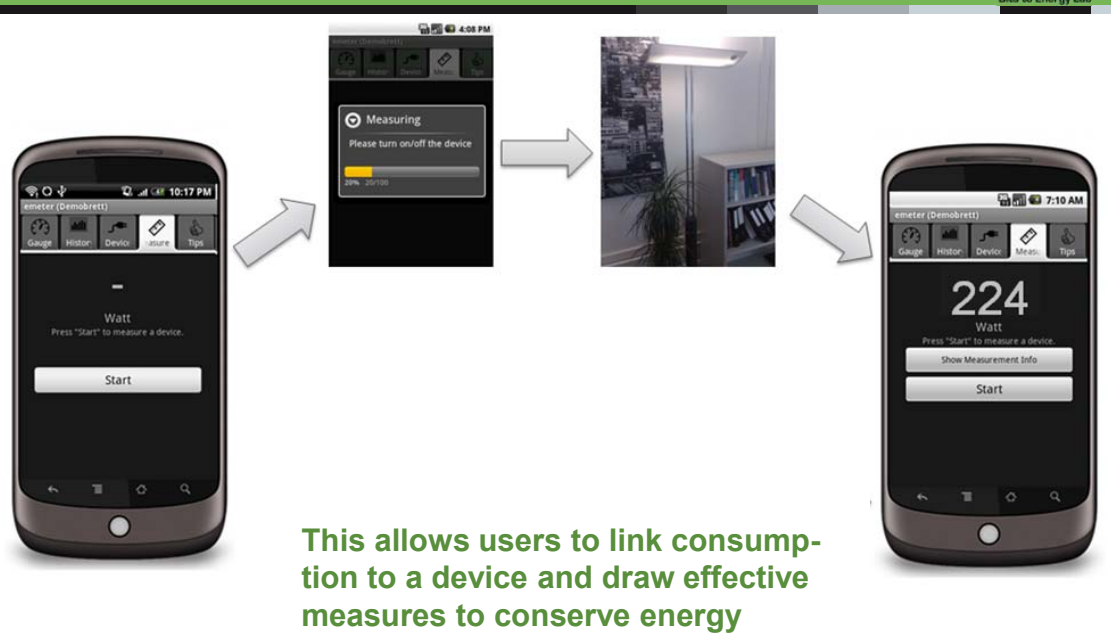




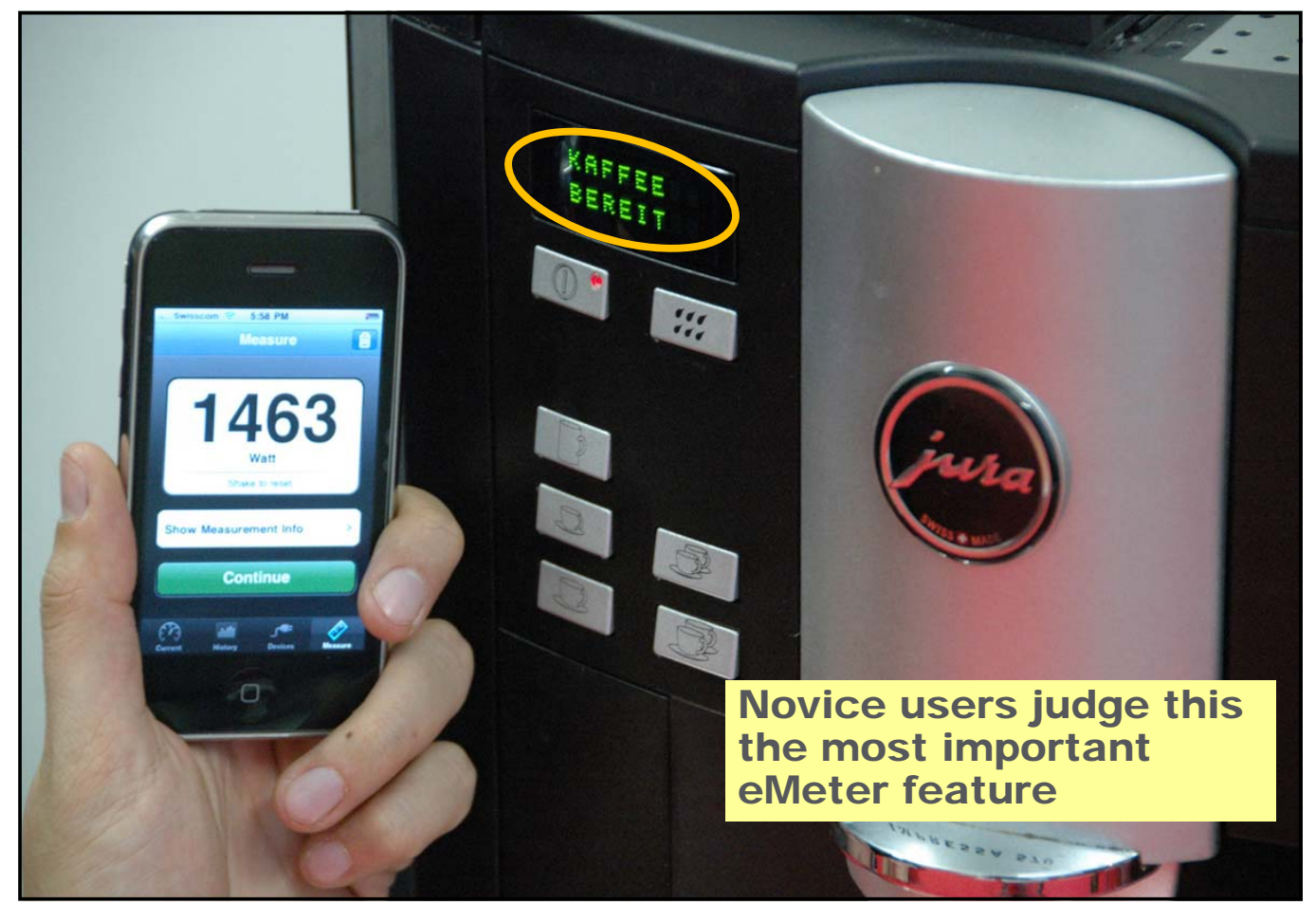

$\rightarrow \quad$ Measured devices can be saved in the device inventory

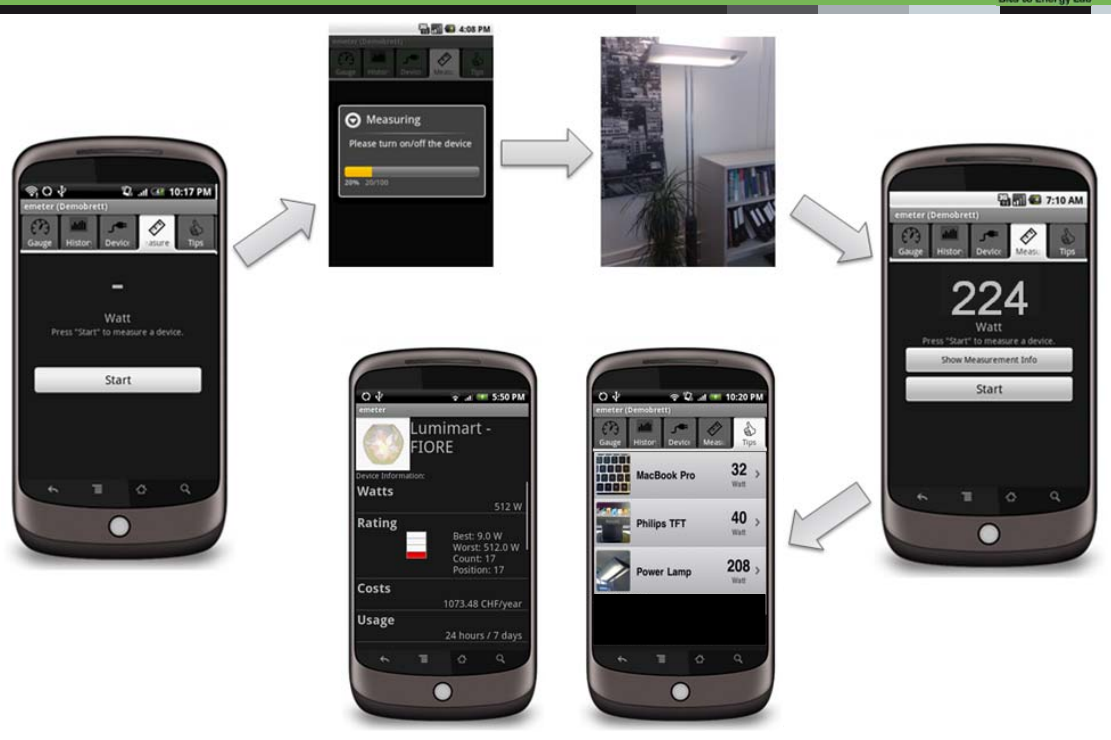



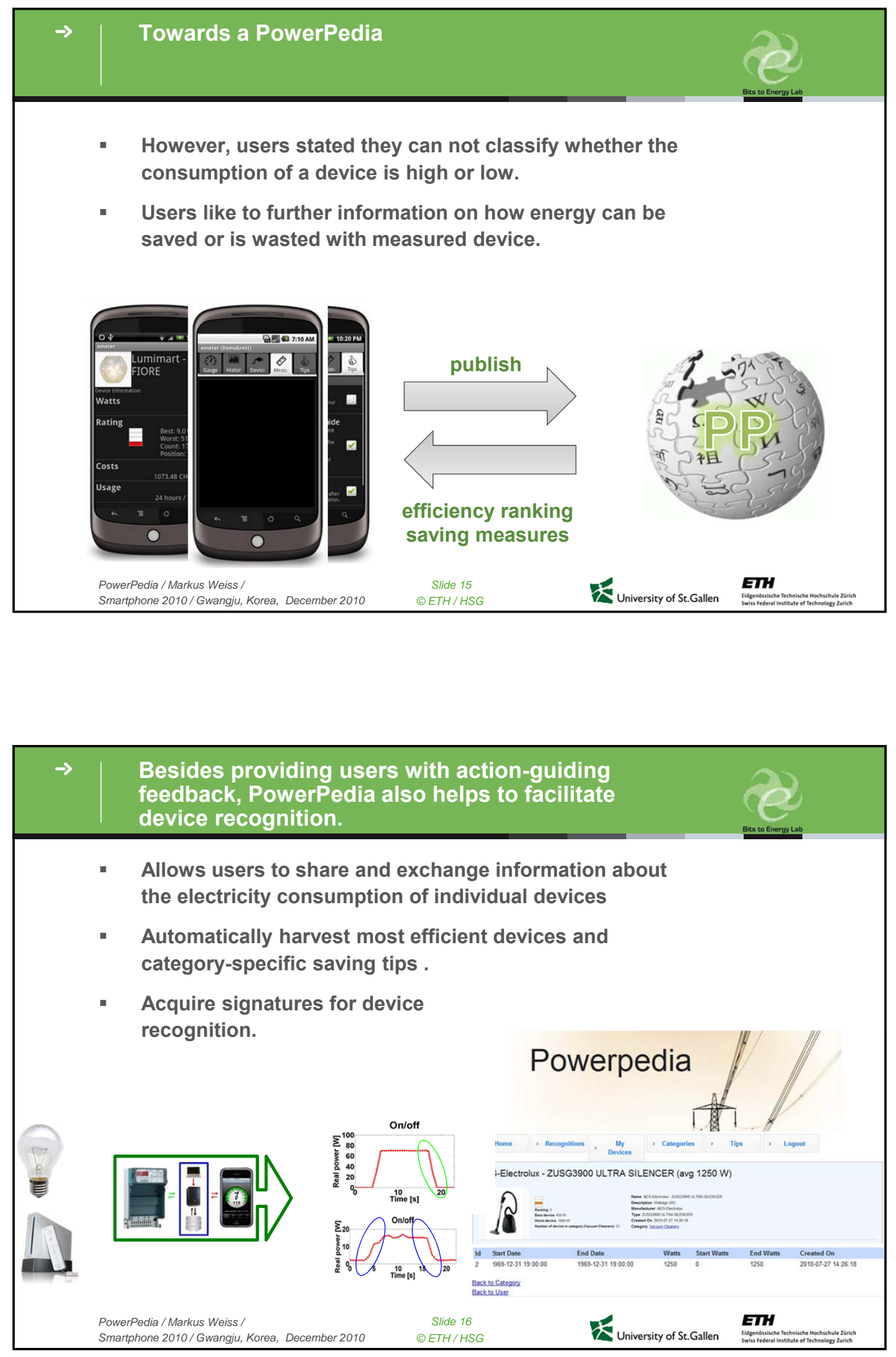


\section{$\rightarrow \quad$ Summary \& Future Work \\ \& Future Work}

- System that allows users to interactively explore their energy consumption aiming at increasing transparency, ...

- User study shows users like more action-guiding information $\rightarrow$ PowerPedia

- At the same time gather DB for load disaggregation algorithm.

- Potential of pattern recognition algorithms applied to the measured load curve to automatically recognize devices.

- Providing feedback to get users in the loop is only the first step to achieve savings. Investigate engagement strategies that involve users over longer time periods.

\section{Thank you}

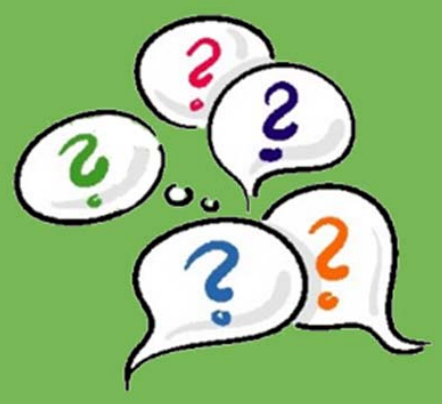

Contact information:

Markus Weiss

Bits to Energy Lab

E-Mail: markus-weiss@ethz.ch
David Abdurachmanov

Fabian Aggeler

Adrian Helfenstein

Adrian Merkle

Wolf Rödiger 


\section{$\rightarrow \quad$ Thank you!}

1

(n)

\section{PowerPedia - A smartphone application}

for community-based electricity feedback

Markus Weiss | markus-weiss@ethz.ch | Office: + 41446328054 |

http://people.inf.ethz.ch/weismark | https://www.im.ethz.ch/people/mweiss/

Institute of Technology Management (ITEM),

University of St. Gallen (HSG),

Department of Management, Technology, and Economics (D-MTEC), and Institute for Pervasive Computing (D-INFK),

ETH Zurich

\section{$\rightarrow \quad$ Further system-related references}

Evaluating Mobile Phones as Energy Consumption Feedback Devices, Markus Weiss, ClaireMichelle Loock, Thorsten Staake, Friedemann Mattern, and Elgar Fleisch, Proc. of Mobiquitous 2010.

Increasing Energy Awareness Through Web-enabled Power Outlets, Markus Weiss and Dominique Guinard, Proc. of MUM 2010.

eMeter: Stromverbrauchsfeedback auf Basis eines Pervasive Energy Monitoring Systems, Markus Weiss, Proc. of Energieinformatik 2010.

Towards a PowerPedia - A collaborative energy encyclopedia, Markus Weiss, Adrian Merkle, Thorsten Staake, and Elgar Fleisch, Proc. of UCSE2010 Workshop at Ubicomp 2010.

Embedding Internet Technology for Home Automation, Matthias Kovatsch, Markus Weiss, Dominique Guinard, Proc. of ETFA 2010.

ICT for Green - How Computers Can Help Us to Conserve Energy!, Friedemann Mattern, Thorsten Staake, and Markus Weiss, Proc. of e-Energy 2010.

Handy feedback: Connecting smart meters with mobile phones, Markus Weiss, Tobias Graml, Thorsten Staake, Friedemann Mattern, Elgar Fleisch. Proc. of MUM 2009.

eMeter: An interactive energy monitor, Markus Weiss, Dominique Guinard, Thorsten Staake, and Wolf Roediger, Adj. Proc. of Ubicomp 2009. 